\title{
Phenotypic and Genotypic Drug Susceptibility Assessment of Mycobacterium bovis Bacillus Calmette-Guérin Clinical Strains
}

This article was published in the following Dove Press journal: Infection and Drug Resistance

\author{
Maria Carolina Sisco ${ }^{1,2}$ \\ Marlei Gomes da Silva' \\ Luciana Distasio de Carvalho ${ }^{3}$ \\ Carlos Eduardo Dias Campos ${ }^{3}$ \\ Paulo Cesar De Souza Caldas ${ }^{3}$ \\ Beatriz Lopez (ID ${ }^{4}$ \\ Claudia Argüelles ${ }^{5}$ \\ Ana Carolina Carvalho (iD ${ }^{6}$ \\ Jacobus de Waard $\mathbb{D}^{7,8}$ \\ Philip Suffys ${ }^{2}$ \\ Rafael Silva Duarte'
}

'Laboratório de Micobactérias, Departamento de Microbiologia Médica, Instituto de Microbiologia Paulo de Góes, Universidade Federal do Rio de Janeiro, Rio de Janeiro, Brazil; ${ }^{2}$ Laboratório de Biologia Molecular Aplicada às Micobactérias, Instituto Oswaldo Cruz, Rio de Janeiro, Brazil; ${ }^{3}$ Laboratório de Referência Nacional Para Tuberculose e Micobacterioses, Centro de Referência Professor Hélio Fraga, Escola Nacional de Saúde Pública, Rio de Janeiro, Brazil; ${ }^{4}$ Instituto Nacional de Enfermedades Infecciosas, Buenos Aires, Argentina; ${ }^{5}$ Instituto Nacional de Producción de Biológicos - ANLIS Carlos G Malbrán, Buenos Aires, Argentina; ${ }^{6}$ Faculdade de Farmácia, Universidade Federal do Rio de Janeiro, Campus Macaé, Rio de Janeiro, Brazil; ${ }^{7}$ Departamento de Tuberculosis, Instituto Autónomo de Biomedicina Dr. Jacinto Convit, Caracas, Venezuela; ${ }^{8}$ One Health Research Group, Facultad de Ciencias de La Salud, Universidad de Las Américas, Quito, Ecuador

Correspondence: Philip Suffys Email psuffys@gmail.com
Purpose: Mycobacterium bovis Bacillus Calmette-Guérin (BCG) is the only vaccine licensed against tuberculosis. Despite the protection offered by the vaccine, in some circumstances children and immunocompromised individuals can develop associated infections, known as BCGitis. Drug susceptibility patterns of BCG clinical strains have rarely been described. We aimed to describe the susceptibility pattern of BCG clinical strains isolated in two different countries.

Methods: We performed culture-based drug susceptibility testing of thirty one BCG strains isolated from patients in Brazil $(n=5,16 \%)$ and Argentina $(n=26,84 \%)$ using the broth micro-dilution method (phenotypic method). Final antibiotic concentrations for susceptibility testing ranged from 0.5 to $16 \mathrm{mg} / \mathrm{L}$ for amikacin, 0.3125 to $10 \mathrm{mg} / \mathrm{L}$ for ethambutol, 0.05 to $1.6 \mathrm{mg} / \mathrm{L}$ for isoniazid and 0.25 to $8 \mathrm{mg} / \mathrm{L}$ for rifampicin, streptomycin and ofloxacin. Additionally, we compared the results with genetic data obtained by whole genome sequencing.

Results: By using the phenotypic method we detected one strain resistant to ethambutol, three strains resistant to rifampicin and one resistant to isoniazid. Additionally, two strains that were phenotypically resistant to both isoniazid and rifampicin carried mutations in the $k a t G$ and $r p o B$ genes simultaneously.

Conclusion: There is evidence of the emergence of BCG-resistant strains isolated from vaccine-related complications. We recommend drug susceptibility testing of the BCG strain causing the infection in order to prevent treatment failure.

Keywords: BCG, mycobacteria, resistance, vaccine

\section{Introduction}

Tuberculosis (TB) remains one of the major causes of mortality worldwide, causing approximately 1.2 million of deaths, with an additional 251,000 deaths among HIVpositive patients. ${ }^{1}$ The BCG vaccine is the only one licensed against tuberculosis and was obtained through continuous passages of a Mycobacterium bovis strain in potato-bile media until attenuation was achieved. ${ }^{2}$ Following its successful application to prevent severe tuberculosis in infants, the strain was sent to multiple laboratories around the world for local production of the vaccine, generating genetically divergent strains, product of the different conditions in which the strains were maintained and processed. ${ }^{3-5}$ Today, BCG vaccines are a family of related strains with described genetic and immunogenic differences ${ }^{6}$ but no superiority regarding protective effect has been demonstrated of one vaccine over the other. ${ }^{7}$ 
Although considered safe, adverse reactions such as local or disseminated infections after vaccination might happen. ${ }^{8}$ At present, there are no standardized guidelines for the management of BCG disease and cases are treated with first-line anti-tuberculosis drugs as isoniazid, ethambutol, rifampicin and streptomycin. However, data on the existence of drug-resistant BCG strains have been reported and demands further investigation of the dimension of this problem by drug susceptibility testing. ${ }^{9,10}$ We therefore verified drug susceptibility of a group of BCG clinical isolates recovered from patients in Brazil and Argentina against the major antibiotics used for treatment of tuberculosis and BCGitis, and compared the results obtained by phenotypical drug susceptibility testing (DST) with data from whole genome sequencing (WGS).

\section{Materials and Methods}

\section{Bacterial Strains and Growth Conditions}

Thirty one BCG clinical isolates were obtained from the bacterial collection of two different laboratories. Five strains $(16 \%)$ were sent by the Laboratório de Referência Nacional de Tuberculose e Micobacterioses Angela Maria Werneck, Centro de Referência Professor Hélio Fraga (CRPHF) in Rio de Janeiro, Brazil. At the time of collection of the strains, the vaccine in use in Brazil was BCG Moreau RDJ. We also received 26 isolates (84\%) from the Instituto Nacional de Enfermedades Infecciosas Dr. Carlos G. Malbrán from Buenos Aires, Argentina, where two vaccines are licensed: BCG Pasteur and BCG Sofia SL222. The isolates had been collected for a period of 15 years (2001-2016) and were maintained in Middlebrook $7 \mathrm{H} 9$ broth (BD Difco) plus 10\% glycerol at $-20^{\circ} \mathrm{C}$. The clinical information of the patients was obtained (when possible) through medical records maintained in each laboratory. Table 1 shows the clinical characteristics of the patients. BCG Moreau RDJ was supplied by Dr. Rafael Silva Duarte of the Laboratory of Mycobacteria at the Federal University of Rio de Janeiro. BCG Pasteur and BCG Sofa SL222 were supplied by Dr. Claudia Argüelles of the National Institute of Biologicals Production of Argentina.

\section{Preparation of Antibiotics and Reagents}

Amikacin, ethambutol, isoniazid, ofloxacin, rifampicin and streptomycin (Sigma-Aldrich) were each dissolved and used according to the manufacturer's instructions. Stock solutions were conserved at $-20^{\circ} \mathrm{C}$. Resazurin sodium salt (Sigma-Aldrich) was prepared as a $0.02 \%$ filtered solution and kept at $4{ }^{\circ} \mathrm{C}$. Final antibiotic concentrations for susceptibility testing ranged from 0.5 to $16 \mathrm{mg} / \mathrm{L}$ for amikacin, 0.3125 to $10 \mathrm{mg} / \mathrm{L}$ for ethambutol, 0.05 to $1.6 \mathrm{mg} / \mathrm{L}$ for isoniazid and 0.25 to $8 \mathrm{mg} / \mathrm{L}$ for rifampicin, streptomycin and ofloxacin.

\section{Drug Susceptibility Assay}

All clinical isolates and the three vaccine strains were submitted to drug susceptibility assays. Quality control of the assays was done by including the strain Mycobacterium tuberculosis H37Rv ATCC 27294. All strains were grown on Middlebrook 7 H10 (BD Difco) and incubated at $37^{\circ} \mathrm{C}$ for at least three weeks.

Two fold-serial dilutions of each drug were prepared in $0.1 \mathrm{~mL}$ of Middlebrook 7H9 broth (BD Difco) supplemented with $10 \%$ oleic acid-albumin-dextrose complex (OADC), casitone, glycerol $(0.05 \%)$ and tween 80 $(0.05 \%)$. To each well, $0.1 \mathrm{~mL}$ of a bacterial suspension adjusted to the density of a McFarland 1 standard was added; then, the plate was incubated at $37^{\circ} \mathrm{C}$; an antibioticfree micro-plate for growth control was also prepared from the bacterial suspension. The bacterial suspension was added in triplicate to the assay plate on the same day as it was prepared. Bacterial growth was confirmed by adding $30 \mu \mathrm{L}$ of resazurin to the control and assay plates; a change of color from blue to pink after 24 hours indicated the reduction of the resazurin and, therefore, cell viability. Minimum inhibitory concentration (MIC) was defined as the lowest concentration of drug that inhibited growth of the microorganism, evidenced by a no reduction of the resazurin (blue color).

\section{Quality Control Assays}

Loss of activity of anti-mycobacterial agents during incubation can lead to false interpretation of results and, in the worst case, cause failures in patient treatment based on non-precise results. We therefore evaluated the stability of anti-mycobacterial drugs during the resazurin micro-plate assay over a period of five weeks. Briefly, $0.1 \mathrm{~mL}$ of a bacterial suspension of $M$. tuberculosis H37Rv ATCC 27294 was added in triplicate to an antibiotic-containing plate prepared as described before and incubated at $37^{\circ} \mathrm{C}$. Then, new suspensions with the same density were added at seven, 14, 21 and 28 days after the plate was prepared 
Table I Clinical Characteristics of the Patients

\begin{tabular}{|c|c|c|c|c|c|}
\hline Country & Strain & Clinical Sample & Age & Comorbidity & Year \\
\hline \multirow[t]{5}{*}{ Brazil } & 4BR & Lymph node & 2 & - & 2016 \\
\hline & $5 B R$ & Lymph node & 2 & - & 2016 \\
\hline & 6BR & Blood culture & 5 months & Milliary TB & 2015 \\
\hline & 7BR & Tracheal aspirate & 5 months & Milliary TB & 2015 \\
\hline & $8 \mathrm{BR}$ & Abscess at the inoculation site & 8 months & - & 2015 \\
\hline \multirow[t]{26}{*}{ Argentina } & IAR & Lymph node & I & - & 2015 \\
\hline & $2 A R$ & Lymph node & I & $\mathrm{HIV+}$ & 2013 \\
\hline & $3 A R$ & Blood culture & - & $\mathrm{HIV+}$ & 2005 \\
\hline & $4 A R$ & Bone biopsy & I & - & 2007 \\
\hline & $5 A R$ & Gastric juice aspirate & 2 & - & 2014 \\
\hline & 6AR & Gastric juice aspirate & 3 & - & 2016 \\
\hline & 7AR & Lymph node & 1 & HIV+ & 2001 \\
\hline & $8 A R$ & Bone marrow & I & $\mathrm{HIV+}$ & 2001 \\
\hline & $9 A R$ & Blood culture & I & $\mathrm{HIV+}$ & 2001 \\
\hline & IOAR & Lymph node & 8 & - & 2003 \\
\hline & IIAR & Lymph node & - & HIV+ & 2005 \\
\hline & I2AR & Lymph node & 4 & HIV- & 2006 \\
\hline & I3AR & Abscess at the inoculation site & I & - & 2009 \\
\hline & I4AR & Lymph node & - & - & 2012 \\
\hline & I5AR & Lymph node & I & $\mathrm{HIV+}$ & 2014 \\
\hline & I6AR & Skin biopsy & - & - & 2014 \\
\hline & I7AR & Abscess at the inoculation site & 2 & - & 2010 \\
\hline & I8AR & Lymph node & I & HIV+ & 2004 \\
\hline & I9AR & Blood culture & - & $\mathrm{HIV+}$ & 2002 \\
\hline & $20 A R$ & Blood culture & - & $\mathrm{HIV+}$ & 2003 \\
\hline & $2 I A R$ & Blood culture & 3 & HIV+ & 2005 \\
\hline & $22 \mathrm{AR}$ & Non identified & I & HIV- & 2005 \\
\hline & $23 A R$ & Bone biopsy & I & HIV- & 2006 \\
\hline & $24 A R$ & Blood culture & I & HIV+ & 2007 \\
\hline & $25 \mathrm{AR}$ & Bone biopsy & - & - & 2013 \\
\hline & $26 \mathrm{AR}$ & Abscess at thumb & I & - & 2015 \\
\hline
\end{tabular}

Note: (-) indicates that related information was not available.

Abbreviations: HIV, human immunodeficiency virus; TB, tuberculosis.

and left incubated at $37^{\circ} \mathrm{C}$. Resazurin was added seven days after each inoculum was added to the plate.

\section{Genome Analysis}

WGS of the 31 strains was performed using the Nextera XT DNA Library preparation kit on an Illumina HiSeq 2500 platform. Quality control of the reads obtained was performed with FastQC program. In order to undercover drug-resistance associated mutations, we performed variant call analysis with the software Snippy v. 4.4 .0 by aligning the reads obtained to reference assemblies of each vaccine obtained from the NCBI. ${ }^{4,11}$ For the analysis of the Brazilian strains, we used the assembly of the Vaccine Moreau RDJ (access GCF_000967285.1) published by Gómes et al. ${ }^{11}$

For the analysis of the Argentinean strains related to patients vaccinated with the Pasteur vaccine, we used the assembly of vaccine Pasteur 1173P2 (access GCF_000009445.1) published by Brosch et al. ${ }^{4}$ For the analysis of the strains from patients vaccinated with BCG Sofia we decided to use the assembly of the vaccine BCG-1 Russia ${ }^{12}$ from which BCG Sofia was delivered, since there is not a whole genome assembly available of this vaccine. The Illumina reads generated were deposited in the Sequence 
Read Archive (SRA) of the NCBI under the Bioproject PRJNA575846.

\section{Results}

\section{Quality Control and Drug Stability Assays}

All drugs maintained the same MIC up until 35 days of incubation, except for ethambutol, that had its value increased two-fold from the fourth week on, reaching a MIC value of five $\mathrm{mg} / \mathrm{L}$ after its fifth week of incubation (Table 2).

\section{Drug Susceptibility Testing of BCG Moreau, BCG Pasteur and BCG Sofia Vaccine Strains}

Recently, broth micro-dilution breakpoints for ethambutol, isoniazid and rifampicin were published by the Clinical and Laboratory Standards Institute (CLSI) for organisms belonging to the Mycobacterium tuberculosis complex. It was stated that a MIC of $4 \mathrm{mg} / \mathrm{L}$ for ethambutol does not correlate with either a susceptible or resistant result in commercial, automated, short incubation broth systems and has not been correlated with clinical response data (Table 3).

For ethambutol, BCG Sofia had the lowest MIC $(0.625 \mathrm{mg} / \mathrm{L})$; however, the control plate presented growth only at 28 days. BCG Moreau had a MIC of $1.25 \mathrm{mg} / \mathrm{L}$, being classified as susceptible. BCG Pasteur had a MIC of $2.5 \mathrm{mg} / \mathrm{L}$ and was classified as inconclusive by the CLSI criteria. Considering isoniazid and rifampicin, the three vaccines had MIC values lower than the CLSI breakpoint. Broth micro-dilution breakpoints for second-line drugs like amikacin, ofloxacin and streptomycin have not been established yet. For amikacin and streptomycin, the three vaccines had MICs of $0.5 \mathrm{mg} / \mathrm{L}$ and $0.25 \mathrm{mg} / \mathrm{L}$, respectively. For ofloxacin, BCG Moreau and Pasteur had a MIC that was two-fold higher than that of BCG Sofia (Table 4).

Table 2 Minimum Inhibitory Concentration of the Antibiotics Tested and Stability Over Time

\begin{tabular}{|l|l|l|l|l|l|}
\hline \multirow{2}{*}{} & \multicolumn{5}{|l}{ Incubation Time (Days) } \\
\cline { 2 - 6 } & $\mathbf{7}$ & $\mathbf{1 4}$ & $\mathbf{2 1}$ & $\mathbf{2 8}$ & $\mathbf{3 5}$ \\
\hline Amikacin & 0.5 & 0.5 & 0.5 & 0.5 & 0.5 \\
Ethambutol & 0.625 & 0.625 & 0.625 & 1.25 & 5 \\
Isoniazid & 0.05 & 0.05 & 0.05 & 0.05 & 0.05 \\
Ofloxacin & 0.25 & 0.25 & 0.25 & 0.25 & 0.25 \\
Rifampicin & 0.25 & 0.25 & 0.25 & 0.25 & 0.25 \\
Streptomycin & 0.25 & 0.25 & 0.25 & 0.25 & 0.25 \\
\hline
\end{tabular}

Note: MIC values are expressed in $\mathrm{mg} / \mathrm{L}$.
Table 3 Breakpoints for Broth Micro-Dilution Method for Mycobacterium tuberculosis Complex Published by the CLSI

\begin{tabular}{|l|l|l|l|}
\hline \multirow{2}{*}{} & \multicolumn{3}{|l|}{ Breakpoints (mg/L) } \\
\cline { 2 - 4 } & Susceptible & Inconclusive & Resistant \\
\hline Ethambutol & $\leq 2$ & 4 & $\geq 8$ \\
Isoniazid & $\leq 0.12$ & - & $\geq 0.25$ \\
Rifampicin & $\leq 1$ & - & $\geq 2$ \\
\hline
\end{tabular}

Table 4 Drug Susceptibility Testing of BCG Vaccines to First and Second-Line Antituberculosis Drugs

\begin{tabular}{|l|l|l|l|l|l|l|}
\hline Vaccine & AMK & EMB & INH & OFX & RIF & STR \\
\hline BCG Moreau & 0.5 & 1.25 & 0.05 & 0.5 & 0.25 & 0.25 \\
BCG Sofia & 0.5 & 0.625 & 0.05 & 0.25 & 0.25 & 0.25 \\
BCG Pasteur & 0.5 & 2.5 & 0.1 & 0.5 & 0.25 & 0.25 \\
\hline
\end{tabular}

Note: MIC values are expressed in $\mathrm{mg} / \mathrm{L}$.

Abbreviations: AMK, amikacin; EMB, ethambutol; INH, isoniazid; OFX, ofloxacin; RIF, rifampicin; STR, streptomycin.

\section{Drug Susceptibility Testing of BCG Clinical Strains}

For ethambutol, we detected one strain related to BCG Sofia to be resistant against this antibiotic. We found two strains resistant to isoniazid in the group of Brazilian strains. In the case of rifampicin, we observed resistant strains in the group related to both the Moreau and the Pasteur vaccine. Figure 1 shows the MIC distribution for all the drugs tested.

Among BCG clinical strains from Brazil, three strains had a MIC for ethambutol two-fold higher than the BCG Moreau vaccine while another strain had a MIC four-fold higher; these strains had MIC values between 2 and $8 \mathrm{mg} / \mathrm{L}$ and could be classified as having intermediate or inconclusive susceptibility. For isoniazid and rifampicin, two strains had a MIC higher than $1.6 \mathrm{mg} / \mathrm{L}$ and $8 \mathrm{mg} / \mathrm{L}$, respectively, being classified as resistant to both antibiotics. One strain had a MIC for amikacin eight-fold higher than the Moreau vaccine (4 mg/L vs $0.5 \mathrm{mg} / \mathrm{L}$ ). For ofloxacin, two strains had a MIC lower than the vaccine $(0.25 \mathrm{mg} / \mathrm{L}$ vs $0.5 \mathrm{mg} / \mathrm{L})$. For streptomycin, all the strains had the same MIC as the vaccine.

In the group of Argentinean strains identified as BCG Sofia, five strains had a MIC for ethambutol higher than the vaccine; one of them had a MIC of $10 \mathrm{mg} / \mathrm{L}$ and was 


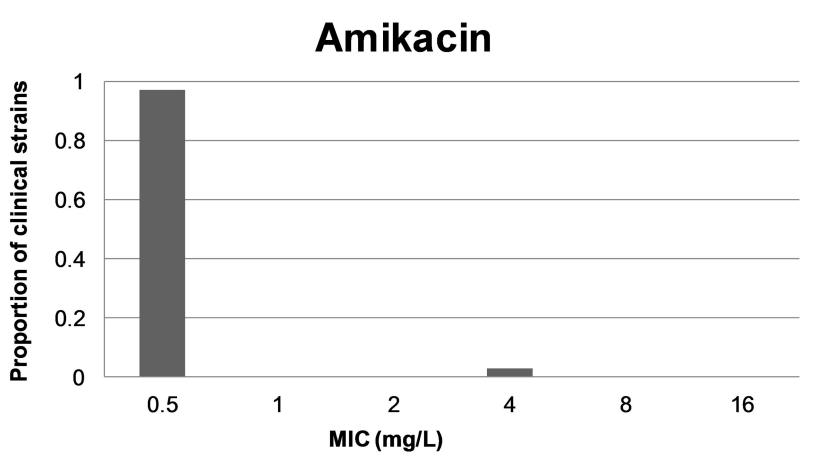

\section{Isoniazid}

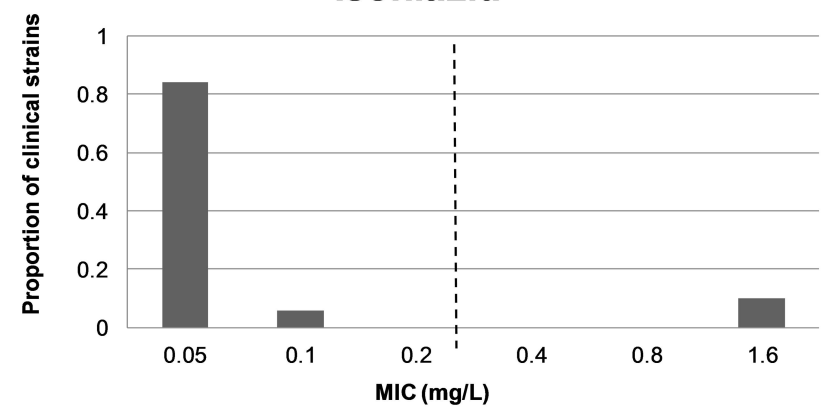

Rifampicin

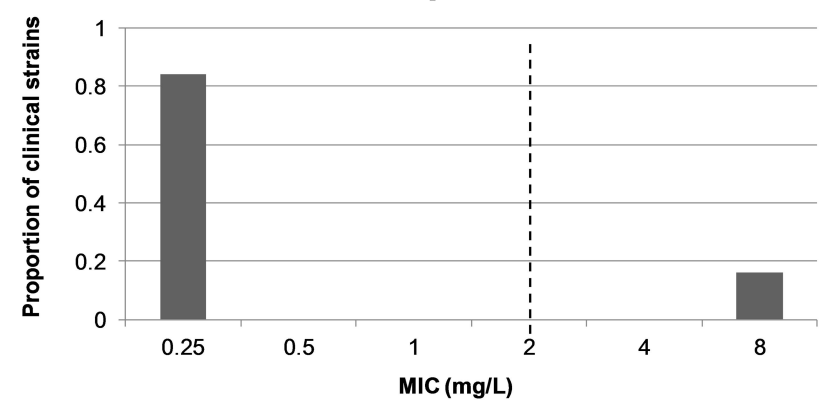

\section{Ethambutol}

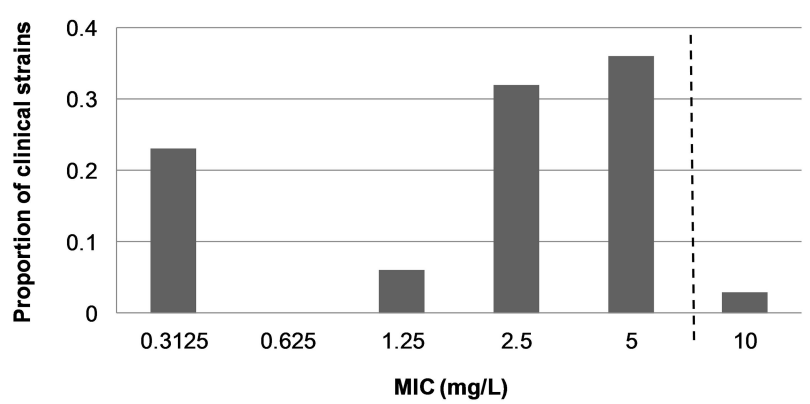

Ofloxacin

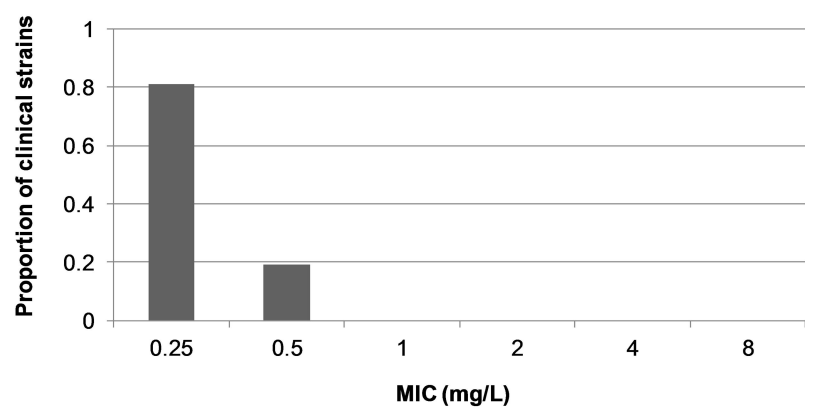

Streptomycin

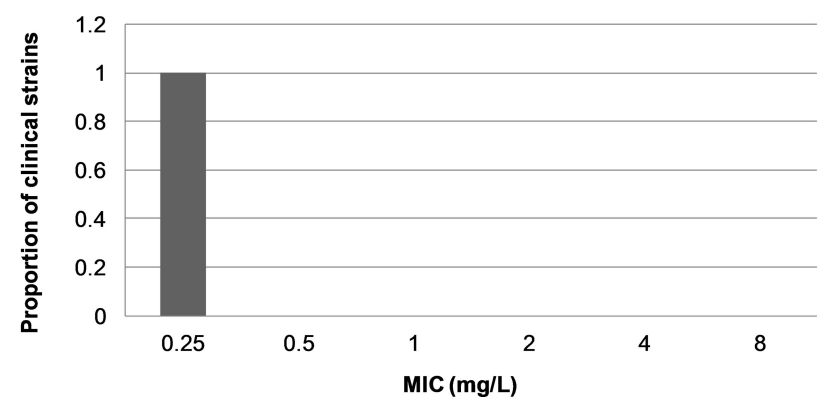

Figure I Minimum inhibitory concentration of BCG clinical strains for all the drugs tested.

Notes: Pointed lines indicate the breakpoint for resistance according to CLSI. For streptomycin, amikacin and ofloxacin the breakpoints for this method have not yet been determined. Abbreviations: MIC, Minimum inhibitory concentration.

considered as resistant. Three strains had a MIC for ofloxacin two-fold higher than the vaccine. For isoniazid, rifampicin and amikacin all the strains had the same MICs as the Sofia vaccine strain.

Finally, in the group of strains from Argentina identified as BCG Pasteur, all the strains had the same MIC value as the vaccine strain for amikacin and streptomycin. Fourteen strains had a MIC for ethambutol between 2.5 and $5 \mathrm{mg} / \mathrm{L}$, hence being classified as having intermediate or inconclusive susceptibility by the CLSI guideline. One strain had a MIC for isoniazid higher than $1.6 \mathrm{mg} / \mathrm{L}$ and was considered resistant. Three strains had a MIC for rifampicin higher than $8 \mathrm{mg} / \mathrm{L}$ and were classified as resistant. All the strains had a MIC for ofloxacin two-fold lower than the vaccine Pasteur.

Table 5 shows the minimal inhibitory concentration of the $\mathrm{BCG}$ clinical strains with the $\mathrm{MIC}_{50}$ and $\mathrm{MIC}_{90}$ statistics meaning the minimum inhibitory concentration at which $50 \%$ and $90 \%$ of the strains are inhibited.

Analyzing the genomes of the BCG clinical strains, we found non-synonymous SNPs in genes associated with resistance to antibiotics in the two Brazilian strains resistant to isoniazid and rifampicin, both strains had 
Table 5 Minimum Inhibitory Concentrations of BCG Clinical Strains Against the Antibiotics Tested

\begin{tabular}{|c|c|c|c|c|}
\hline \multicolumn{5}{|c|}{ BCG Moreau-Related Clinical Strains } \\
\hline & Range & $\mathrm{MIC}_{50}$ & MIC $_{90}$ & Mode \\
\hline Amikacin & $0.5-4$ & 0.5 & 0.5 & 0.5 \\
\hline Ethambutol & $1.25-5$ & 2.5 & 2.5 & 2.5 \\
\hline Isoniazid & $0.05-1.6$ & 0.1 & 1.6 & $0.1-1.6$ \\
\hline Ofloxacin & $0.25-0.5$ & 0.5 & 0.5 & 0.5 \\
\hline Rifampicin & $0.25-8$ & 0.25 & 8 & 0.25 \\
\hline Streptomycin & 0.25 & 0.25 & 0.25 & 0.25 \\
\hline \multicolumn{5}{|c|}{ BCG Sofia-Related Clinical Strains } \\
\hline & Range & $\mathrm{MIC}_{50}$ & $\mathrm{MIC}_{90}$ & Mode \\
\hline Amikacin & 0.5 & 0.5 & 0.5 & 0.5 \\
\hline Ethambutol & $0.3125-10$ & 2.5 & 5 & $2.5-5$ \\
\hline Isoniazid & 0.05 & 0.05 & 0.05 & 0.05 \\
\hline Ofloxacin & $0.25-0.5$ & 0.25 & 0.5 & $0.25-0.5$ \\
\hline Rifampicin & 0.25 & 0.25 & 0.25 & 0.25 \\
\hline Streptomycin & 0.25 & 0.25 & 0.25 & 0.25 \\
\hline \multicolumn{5}{|c|}{ BCG Pasteur-Related Clinical Strains } \\
\hline & Range & $\mathrm{MIC}_{50}$ & $\mathrm{MIC}_{90}$ & Mode \\
\hline Amikacin & 0.5 & 0.5 & 0.5 & 0.5 \\
\hline Ethambutol & $0.3125-5$ & 2.5 & 5 & 5 \\
\hline Isoniazid & $0.05-1.6$ & 0.05 & 0.05 & 0.05 \\
\hline Ofloxacin & 0.25 & 0.25 & 0.25 & 0.25 \\
\hline Rifampicin & $0.25-8$ & 0.25 & 8 & 0.25 \\
\hline Streptomycin & 0.25 & 0.25 & 0.25 & 0.25 \\
\hline
\end{tabular}

Note: MIC values are expressed in $\mathrm{mg} / \mathrm{L}$.

Abbreviation: $\mathrm{MIC}_{50}$ and $\mathrm{MIC}_{90}$, minimum inhibitory concentration at which $50 \%$ and $90 \%$ of the strains are inhibited.

nsSNP in the kat $G$ and $r p o B$ genes simultaneously (kat $G$ $\mathrm{S} 293 \mathrm{~T}$ and $r p o B$ D359V). Another strain in this group had a deletion of three nucleotides in rрoB but was sensitive to rifampicin by the phenotypical method. Mutations associated with resistance to amikacin, ethambutol, ofloxacin and streptomycin were not found during the analysis.

In the phenotypically resistant strains found in the Argentinean group, no mutations associated with any antibiotic were detected.

\section{Discussion}

Loss of activity of anti-mycobacterial agents during incubation can lead to false interpretation of results and, in the worst case, cause failures in patient treatment based on non-precise results. We therefore evaluated the stability of anti-mycobacterial drugs during the resazurin micro-plate assay over a period of five weeks. All drugs maintained the same MIC up until 35 days of incubation, except for ethambutol. An extensive evaluation of stability of several anti-mycobacterial drugs was recently reported by Schoutrop et al, $^{13}$ observing at least a four-fold increase in the MIC for rifampicin and streptomycin when using commercial micro-plate assay in cation adjusted Mueller Hinton Broth (CAMHB), incubated at $37^{\circ} \mathrm{C}$ for fourteen days and using the reference strain Mycobacterium avium subsp. hominissuis IWGMT49. Different from their results, we observed that stability of these drugs is maintained when using 7H9 Middlebrook Broth supplemented with OADC. Schoutrop et al also demonstrated that dilution of the drugs in $7 \mathrm{H} 9$ Broth with OADC and incubation at $37^{\circ} \mathrm{C}$ reduced concentration of isoniazid and rifampicin in $7 \mathrm{H} 9$ media (54\% and $92 \%$ respectively) after seven days, while our results show that the activity of both drugs remains unaffected for at least five weeks. Perhaps, these discrepancies are due to the presence of some residual anti-mycobacterial activity of secondary products during degradation of the drug, the latter suggested by the authors. Griffith and Bodily, ${ }^{14}$ described the loss of half of the activity of ethambutol dispensed on solid agar $7 \mathrm{H} 10$ media and maintained at $37^{\circ} \mathrm{C}$ after two to four days of incubation; these results are in agreement with our present observations. Our results show that, using $7 \mathrm{H} 9$ broth media, the activity of ethambutol remains unaffected until 21 days but that for Mycobacterium species that require longer incubation times, interpretation of results using this drug needs caution. Under our experimental conditions, amikacin, isoniazid, ofloxacin, rifampicin and streptomycin were stable for at least 35 days.

We observed a lack of agreement between drug resistance as determined by phenotypical DST and the presence of genetic modifications that have been associated with drug resistance. This inconsistency has been reported before and is a major problem for patient treatment using genetic-based procedures for drug resistance without confirmation by conventional DST. ${ }^{15-17}$ This phenomenon could be related to the existence of unknown resistance mechanisms like non described mutations in unknown drug-associated genes and contribution of efflux pumps. ${ }^{18}$ 
Strains harboring drug-associated mutations but being phenotypically sensitive have been described for M. tuberculosis strains in which MICs for streptomycin, ethambutol or rifampicin were increased only slightly, ${ }^{15}$ this might be due to difficulties inherent to the broth micro-dilution technique and could be more evident in front of higher antibiotic concentrations during the phenotypic assay.

Another factor to consider is that almost all drug susceptibility testing procedures, either based on culture or genotyping-based procedures were performed on strains of M. tuberculosis and that few data exist on DST in BCG clinical strains. Resistance to both isoniazid and ethionamide was previously observed in strains of BCG Danish and BCG Connaught. ${ }^{19,20}$ Intermediate susceptibility of BCG Danish had been addressed by the Statens Serum Institut and is now specified in the leaflet of the vaccine; nonetheless, the WHO states that there is no need of a change of vaccine policy. ${ }^{21}$ Intrinsic resistance to isoniazid was observed by Hesseling et al in an isolate from a six-month-old HIV-positive child with axillar adenitis that had been vaccinated with BCG Danish vaccine; the strain later acquired resistance to rifampicin during the child's treatment. ${ }^{9}$ In a study performed in the Netherlands, five strains isolated in children with lymphadenitis had a MIC higher $(0.5 \mu \mathrm{g} / \mathrm{mL})$ than that observed for the vaccine $(0.1-0.2 \mu \mathrm{g} / \mathrm{mL}){ }^{22}$ Finally, in Brazil, a BCG strain isolated from an immunocompromised child with lymphadenitis was intrinsically resistant to isoniazid and later became resistant to rifampicin, sequence analysis demonstrated the $\mathrm{S} 315 \mathrm{~T}$ mutation in katG gene and $\mathrm{D} 516 \mathrm{~V}$ mutation in $r p o B$ gene. ${ }^{10}$

\section{Conclusion}

Infections caused by vaccination with BCG are scarce and when diagnosed and treated early, there is a high probability of positive outcome. However, our report adds information on cases with intrinsic or acquired resistance to antibiotics and highlights the need for drug susceptibility screening of the strain before or during patient treatment. Also, we emphasize the urgency for standardization of second-line drugs' breakpoints for broth micro-dilution method in view of the emergence of first-line drug-resistant strains.

\section{Acknowledgments}

We thank Dr. Leila Mendonça-Lima for supplying the DNA of the vaccine BCG Moreau. We also acknowledge the sequencing platform of Fiocruz (RPT01J) and Ricardo
Junqueira for assistance in the preparation of libraries, Kamila Chagas Peronni from the Laboratory of Molecular Genetics and Bioinformatics from the Regional Center of Haemotherapy and Professor Valdes Bollela from the School of Medicine, São Paulo University in Ribeirão Preto.

\section{Sponsorships}

This study was financed in part by the Coordenação de Aperfeiçoamento de Pessoal de Nível Superior - Brazil (CAPES) - Finance Code 001. The authors also acknowledge the support of Conselho Nacional de Desenvolvimento Científico e Tecnológico - CNPq and Fundação Carlos Chagas Filho de Amparo à Pesquisa do Estado do Rio de Janeiro (FAPERJ). PNS was supported by CNPq grant PQ 310,418/2016-0.

\section{Disclosure}

Dr Maria Carolina Sisco report grants from Coordenação de Aperfeiçoamento de Pessoal de Nível Superior - Brazil (CAPES), grants from Conselho Nacional de Desenvolvimento Científico e Tecnológico - CNPq, during the conduct of the study. The authors report no other conflicts of interest in this work.

\section{References}

1. Global tuberculosis report. World Health Organization: 2019.

2. Calmette A. Preventive vaccination against tuberculosis with BCG. Proc R Soc Med. 1931;85-94.

3. Behr MA. BCG - Different strains, different vaccines? Lancet Infect Dis. 2002;2(2):86-92. doi:10.1016/S1473-3099(02)00182-2

4. Brosch R, Gordon SV, Garnier T, et al. Genome plasticity of BCG and impact on vaccine efficacy. Proc Natl Acad Sci U S A. 2007;104 (13):5596-5601. doi:10.1073/pnas.0700869104

5. Tran V, Liu JUN, Behr MA. BCG vaccines. Microbiol Spectrum. 2014;2(1):1-11. doi:10.1128/microbiolspec.MGM2-0028-2013

6. Zhang L, Ru HW, Chen FZ, et al. Variable virulence and efficacy of BCG vaccine strains in mice and correlation with genome polymorphisms. Mol Ther. 2016;24(2):398-405. doi:10.1038/ mt.2015.216

7. Barker LF. The Immunological Basis for Immunization Series. Module 5: Tuberculosis. Geneva: World Health Organization; 2011:29.

8. Talbot EA, Perkins MD, Silva SF, Frothingham R. Disseminated bacille Calmette-Guérin disease after vaccination: case report and review. Clin Infect Dis. 1997;24(6):1139-1146. doi:10.1086/ 513642

9. Hesseling AC, Schaaf HS, Victor T, et al. Resistant Mycobacterium bovis Bacillus Calmette-Guérin disease: implications for management of Bacillus Calmette-Guérin Disease in human immunodeficiency virus-infected children. Pediatr Infect Dis J. 2004;23(5):476-479. doi:10.1097/01.inf.0000126593.21006.ac

10. Diniz LM, Guimarães T, Oliveira M, Pinto JA, Miranda SS. Lymphadenitis caused by infection with an isoniazid- and rifampinresistant strain of Mycobacterium bovis BCG in an infant with IFN- $\gamma$ / IL-12 pathway defect. J Bras Pneumol. 2014;40(2):188-192. doi:10.1590/S1806-37132014000200014 
11. Gomes LHF, Otto TD, Vasconcellos ÉA, et al. Genome sequence of Mycobacterium bovis BCG Moreau, the Brazilian vaccine strain against tuberculosis. $J$ Bacteriol. 2011;193(19):5600-5601. doi: 10.1128/JB.05827-11

12. Sotnikova EA, Shitikov EA, Malakhova MV, et al. Complete genome sequence of Mycobacterium bovis strain BCG-1 (Russia). Genome Announc. 2016;4(2):2007-2008.

13. Schoutrop ELM, Brouwer MAE, Jenniskens JCA, et al. The stability of antimycobacterial drugs in media used for drug susceptibility testing. Diagn Microbiol Infect Dis. 2018;92(4):305-308. doi:10.1016/j.diagmicrobio.2018.06.015

14. Griffith ME, Bodily HL. Stability of antimycobacterial drugs in susceptibility testing. Antimicrob Agents Chemother. 1992;36 (11):2398-2402. doi:10.1128/AAC.36.11.2398

15. Ruesen C, Riza AL, Florescu A, et al. Linking minimum inhibitory concentrations to whole genome sequence-predicted drug resistance in Mycobacterium tuberculosis strains from Romania. Sci Rep. 2018;8(1):9676. doi:10.1038/s41598-018-27962-5

16. Williamson DA, Roberts SA, Bower JE, et al. Clinical failures associated with rpoB mutations in phenotypically occult multidrug-resistant Mycobacterium tuberculosis. Int J Tuberc Lung Dis. 2012;16(2):216-220. doi:10.5588/ijtld.11.0178
17. Ho J, Jelfs P, Sintchencko V. Phenotypically occult multidrug-resistant Mycobacterium tuberculosis: dilemmas in diagnosis and treatment. $J$ Antimicrob Chemother. 2013;68 (12):2915-2920. doi:10.1093/jac/dkt284

18. Kanji A, Hasan R, Hasan Z. Efflux pump as alternate mechanism for drug resistance in Mycobacterium tuberculosis. Indian J Tuberc. 2019;66(1):20-25. doi:10.1016/j.ijtb.2018.07.008

19. Ritz N, Tebruegge M, Connell TG, Sievers A, Robins-Browne R, Curtis N. Susceptibility of Mycobacterium bovis BCG vaccine strains to antituberculous antibiotics. Antimicrob Agents Chemother. 2009;53 (1):316-318. doi:10.1128/AAC.01302-08

20. Watts MR, Taylor PC, Sintchenko V, et al. Editor's choice: implications of isoniazid resistance in Mycobacterium bovis Bacillus Calmette-Guérin used for immunotherapy in bladder cancer. Clin Infect Dis. 2011;52(1):86-88. doi:10.1093/cid/ciq002

21. WHO Discussion on the Improvement of the Quality Control of BCG Vaccines. França: World Health Organization; 2005.

22. Arend SM, Van Soolingen D. Low level INH-resistant BCG: a sheep in wolf's clothing? 2011;89-93. doi:10.1093/cid/ciq016

\section{Publish your work in this journal}

Infection and Drug Resistance is an international, peer-reviewed openaccess journal that focuses on the optimal treatment of infection (bacterial, fungal and viral) and the development and institution of preventive strategies to minimize the development and spread of resistance. The journal is specifically concerned with the epidemiology of antibiotic resistance and the mechanisms of resistance development and diffusion in both hospitals and the community. The manuscript management system is completely online and includes a very quick and fair peerreview system, which is all easy to use. Visit http://www.dovepress.com/ testimonials.php to read real quotes from published authors. 